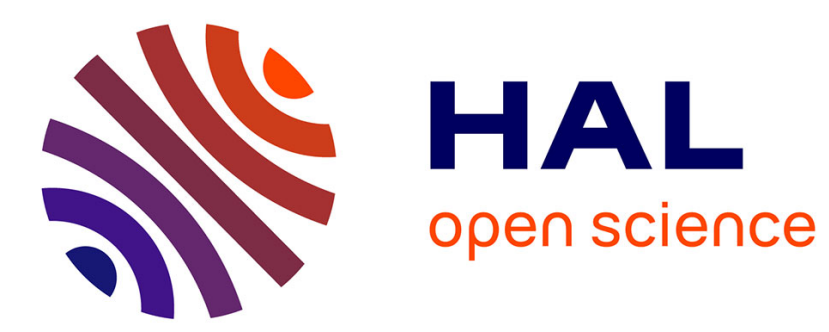

\title{
Il processo di Cremuzio Cordo in Dio LVII, 24, 2-4
}

Martina Bono

\section{To cite this version:}

Martina Bono. Il processo di Cremuzio Cordo in Dio LVII, 24, 2-4. Archimède : archéologie et histoire ancienne, 2016, 3, pp.218-227. hal-01587269

\section{HAL Id: hal-01587269 \\ https://hal.science/hal-01587269}

Submitted on 14 Sep 2017

HAL is a multi-disciplinary open access archive for the deposit and dissemination of scientific research documents, whether they are published or not. The documents may come from teaching and research institutions in France or abroad, or from public or private research centers.
L'archive ouverte pluridisciplinaire HAL, est destinée au dépôt et à la diffusion de documents scientifiques de niveau recherche, publiés ou non, émanant des établissements d'enseignement et de recherche français ou étrangers, des laboratoires publics ou privés. 


\section{DOSSIER THÉMATIQUE : DES FOSSÉS ET DES REMPARTS. ENCEINTES ET SITES FORTIFIÉS DU RHIN SUPÉRIEUR ENTRE PROTOHISTOIRE ET MOYEN ÂGE}

\section{Olivier BUCHSENSCHUTZ}

Avant-propos. Des enceintes en terre anhistoriques à Google Earth

8 Lizzie SCHOLTUS

Histoire de la recherche dans le bassin de Saint-Dié-des-Vosges

20 Maxime WALTER

Les sites de hauteur du massif vosgien. Actualisation des données et modalités d'implantation

37 Jean-Jacques SCHWIEN

Chateaux et enceintes des Vosges du Nord. Topographie et longue durée

49 Anne-Marie ADAM

La palissade dans tous ses états : I'enclos du Britzgyberg (Illfurth, Haut-Rhin) et autres aménagements palissadés dans les habitats du premier âge du Fer

60 Clément FÉLIU

L'enceinte inférieure du Frankenbourg (67) et les remparts à poteaux frontaux de la fin de l'âge du Fer dans l'espace du Rhin supérieur. Pour une révision de la typologie des Pfostenschlitzmauern

74 Jacky $\mathrm{KOCH}$ et Thomas FISCHBACH

Enceintes de hauteur en pierres et formes « primitives » de châteaux ? L'exemple du Bernstein

87 Adrien VUILLEMIN

Les enceintes urbaines en moyenne Alsace (1200-1850)

102 Jean-François PININGRE

Les enceintes de l'âge du Bronze et du premier âge du Fer en Franche-Comté. Un bilan des recherches

124 Clément FÉLIU et Jean-Jacques SCHWIEN

Conclusion. Nouvelles perspectives sur les enceintes du Rhin supérieur

\section{ACTUALITÉ DE LA RECHERCHE : ARCHÉOLOGIE DES RÉSEAUX}

\section{Claire CAMBERLEIN}

Les réseaux en archéologie : approche historiographique et interdisciplinaire

135 Thomas HUTIN

Lieux d'échanges et espaces publics en Gaule à La Tène finale

150 Streeve GENTNER

Économie du fer et voies de communication, de l'abattage du minerai à la distribution

du métal : I'exemple du nord de la Forêt-Noire au Ve siècle av. J.-C.

169 Loup BERNARD et Rémy WASSONG

Du Danemark au Fossé rhénan. Un siècle d'analyse des voies de communications protohistoriques : évolution des méthodes et mise en commun des données

184 Steeve GENTNER et Rémy WASSONG

Conclusion. L'archéologie des réseaux : une thématique aux multiples facettes

\section{VARIA}

187 Fábio VERGARA CERQUEIRA

To march in phalanx, to jump with weights, to tread the grapes, to knead the bread. What is the aulos for?

206 Hermann AMON

Les supra-commandements comme solution à la crise militaire du III siècle de l'Empire romain sous Philippe l'Arabe et Gallien

218 Martina BONO

Il processo di Cremuzio Cordo in Dio LVII, 24, 2-4

\section{LA CHRONIQUE D'ARCHIMÈDE}

228 Frédéric COLIN (éd.)

La Chronique d'Archimède. Bilan des activités scientifiques 2015-2016 de I'unité mixte de recherche 7044 


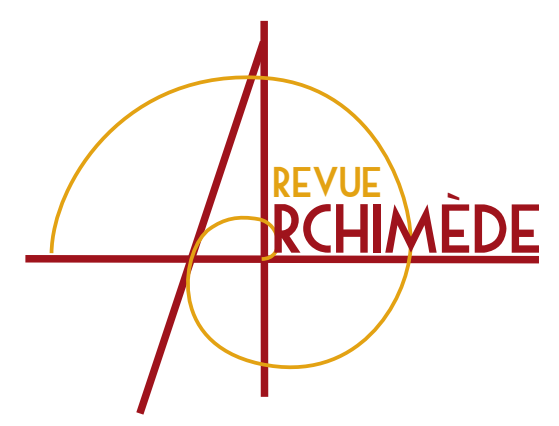

\section{IL PROCESSO DI CREMUZIO CORDO IN DIO LVII, 24, 2-4}

Martina BONO

Doctorante en sciences de l'Antiquité (Histoire romaine) Université de Pavie et Université de Haute-Alsace

\section{RÉSUMÉ}

martina.bono01@universitadipavia.it

Dans cet article, l'auteur se focalise sur les témoignages qui concernent le procès contre Cremutius Cordus, qui fut accusé de maiestate en 25 ap. J.-C. Parmi les sources parvenues, il y a le récit de Dion Cassius, qui n'a pas été mis en valeur par les spécialistes puisque le témoignage de Tacite est considéré comme le plus fiable et exhaustif. L'article propose une analyse du texte de cet historien (Dion Cassius LVII, 24, 2-4), afin d'expliquer ce qu'il a voulu dire à travers des expressions peu claires et très concises. Le but de cette étude est aussi bien de clarifier le déroulement du procès de Cremutius, que de réfléchir sur la valeur documentaire de l'ouvre de Dion Cassius.

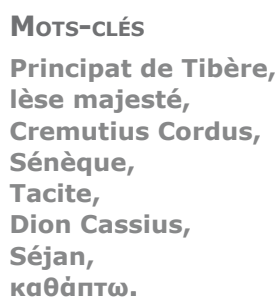

Nel presente articolo, l'autrice si concentra sulle testimonianze riguardanti il caso del senatore Cremuzio Cordo, autore di Annales, accusato nel 25 d.C. de maiestate. Tra le testimonianze possedute, quella trasmessa dallo storico bitinico Cassio Dione (LVII, 24, 2-4) ha ricevuto poca attenzione da parte degli studiosi, poiché considerate più esaustive le informazioni riferite sulla vicenda da Tacito (Ann. IV, 34-35). Attraverso una disamina approfondita della notizia dionea ci si propone di spiegare alcuni luoghi del testo che, poco perspicui a causa della loro sinteticità espressiva, non sono stati oggetto di un'adeguata esegesi. Pertanto, ci si propone sia di chiarire alcuni aspetti della vicenda di Cremuzio, sia di rimarcare il valore documentario dell'opera di Cassio Dione.

$$
\begin{aligned}
& \text { Parole chiave } \\
& \text { Principato di Tiberio, } \\
& \text { laesa maiestas, } \\
& \text { Cremuzio Cordo, } \\
& \text { Seneca, } \\
& \text { Tacito, } \\
& \text { Cassio Dione, } \\
& \text { Seiano, } \\
& \text { käántw. }
\end{aligned}
$$

In this article, the author focuses upon the testimonies on the trial of the senator Cremutius Cordus, author of Annales, who was charged in $A D$ 25 with the accusation de majestate. Among the ancient sources, the historian Cassius Dio has not been considered worthy of special attention. Dio's concise style and elliptic wording has conveyed the idea that he cannot furnish much additional information to what already said by Tacitus in his historical work (Ann. IV, 34-35). However, Dio's text may give us an insight into a plurality of accusations de maiestate against Cremutius, whereas, according to Tacitus, Cremutius was brought into a trial "only" for having praised Brutus and Cassius. Through a detailed analysis of the testimony of Dio, we will try both to clarify some aspects of the Cremutius-case, and to cast light on the documentary relevance of the passage in Dio.
KeYWORDS

Tiberius' Principate, lex de majestate, Cremutius Cordus, Seneca, Tacitus, Cassius Dio, Sejanus, кaӨántw. 
Poco numerose e difformi nel contenuto, le fonti riguardanti la vicenda giudiziaria e I'opera letteraria dello storico e senatore Cremuzio Cordo sono, anche cronologicamente, piuttosto circoscritte: si può tracciare una linea che va dal I secolo con Seneca Padre (Suas. VI, 19; 23), Seneca (Cons. Marc. 1,$3 ; 22,4 ; 26,5)$, Plinio il Vecchio (N.H. X, 74; N.H. XVI, 108) e Quintiliano [1] (Inst. X, 1, 104), al II secolo con Tacito (Ann. IV, 34-35) e Svetonio (Aug. 35, 2; Tib. 61, 3), fino a giungere al III secolo con Cassio Dione. Proprio quest'ultimo, il quale ha lasciato in uno dei sui libri sul regno Tiberio (il LVII) la notizia del processo de maiestate contro Cremuzio Cordo è spesso stato sottovalutato dalla critica; il testo della notizia dionea è il seguente (Dio LVII, 24, 2-4):

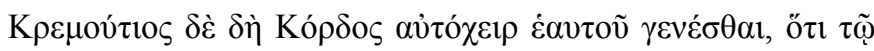

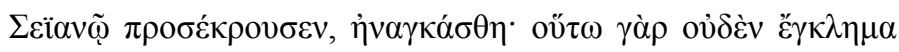

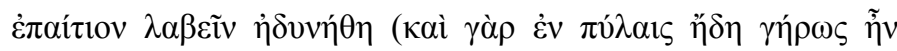

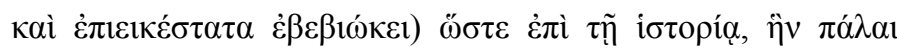

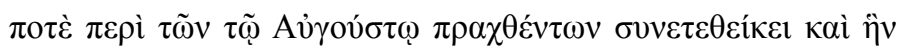

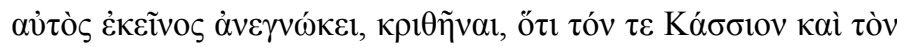

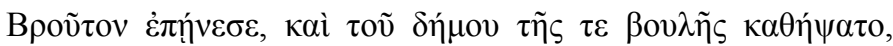

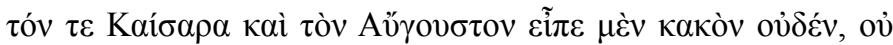

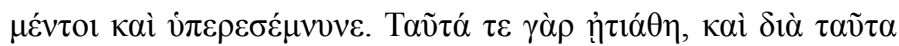

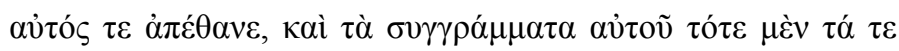

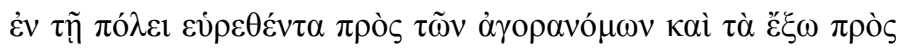

[1] Vd. CANFORA 1993, p. 234-236.

[2] Dio, LVII, 24: «Cremuzio Cordo, poiché era venuto in conflitto con Seiano, fu costretto a togliersi la vita. A tal punto era vissuto in modo irreprensibile ed era già alle soglie della vecchiaia che infatti nessuna accusa gli poteva essere imputata contro, cosicché fu processato per l'opera storica, la quale aveva composto in passato riguardo alle gesta di Augusto e che questo stesso aveva letto, sia per aver lodato Bruto e Cassio, per aver aggredito il popolo e il Senato e per il fatto che aveva parlato di Cesare e di Augusto non in modo negativo, ma certamente non li aveva neppure riempiti di lodi. Queste dunque furono le cose per le quali fu accusato, e proprio per queste stesse egli morì, e i suoi scritti quanti allora si trovavano nella città furono bruciati dagli edili, dai magistrati di ciascun luogo quanti si trovavano al di fuori di essa, mentre in seguito furono pubblicati nuovamente; alcuni e soprattutto sua figlia Marcia li

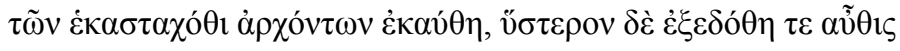

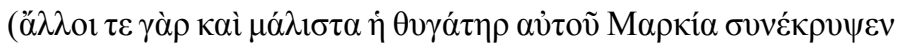

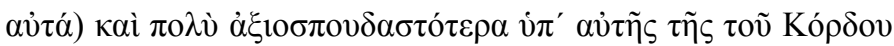

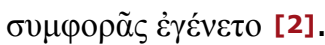

La testimonianza di Cassio Dione ha il sapore di una riflessione, di una ricerca. Il nostro autore analizza il fatto storico, indagando motivazioni e conseguenze:

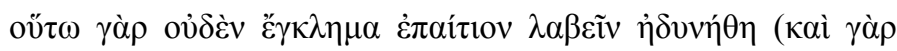

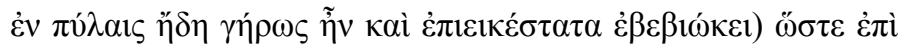

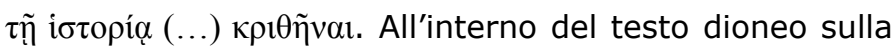
vicenda dello storico Cremuzio Cordo sono da rilevare, visto lo spessore di significato, a tratti allusivo- cosa che invece ha spinto i più a tralasciarne una specifica disamina-, almeno tre luoghi in qualità di punti chiave:

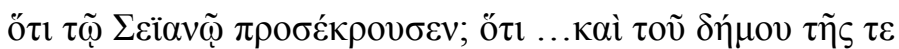

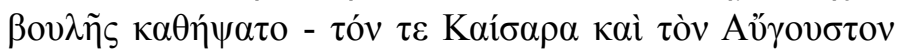

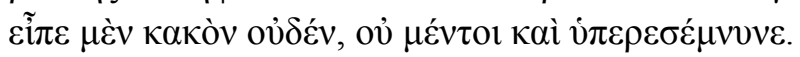

Innanzitutto, è da mettere in rilievo il fatto che Cassio Dione attribuisca al prefetto del pretorio Lucio Elio Seiano il ruolo di mandante dell'accusa de maiestate contro Cremuzio Cordo, in quanto era venuto in conflitto con lui ( $\pi \rho \circ \sigma \varepsilon ́ \kappa \rho о \nu \sigma \varepsilon v)$. La notizia collima con quanto testimoniato da Seneca (Sen. Marc. 22,4) [3], il quale, come intimo di Marcia, la figlia dello stesso storico imputato cui dedica una Consolatio, deve aver attinto a informazioni

avevano nascosti e furono molto degni di interesse per la sventura stessa di Cordo».

[3] Propone illud acerbissimum tibi tempus, quo Seianus patrem tuum clienti suo Satrio Secundo congiarium dedit. Irascebatur illi ob unum aut alterum liberius dictum, quod tacitus ferre non potuerat Seianum in cervices nostras ne imponi quidem, sed escendere. Decernebatur illi statua in Pompei theatro ponenda, quod exustum Caesar reficiebat; exclamavit Cordus tunc vere theatrum perire. Trad. it.: «Richiama alla memoria quel tempo per te dolorosissimo, in cui Seiano diede in dono al suo cliente Satrio Secondo tuo padre. Si era irritato con lui per una o due battute più franche, poiché non aveva potuto sopportare in silenzio non tanto che Seiano fosse imposto, ma che salisse sulle nostre teste. Si decretava per lui l'erezione di una statua nel teatro di Pompeo, che Cesare restaurava dopo un incendio; Cordo esclamò che allora veramente il teatro andava in rovina». 
di prima mano e proprio per tale aspetto dotate di un grado maggiore di attendibilità. Diversamente Tacito, che è considerato la fonte principale per la conoscenza del caso di Cremuzio Cordo, sembra fare riferimento al prefetto del pretorio soltanto implicitamente, attraverso la menzione dei suoi clientes Satrio Secondo e Pinario Natta, quali delatori e subscriptores [4]. Infine, Svetonio è il solo a fare di Tiberio il vero promotore del processo [5], annoverando, in modo anonimo, il caso dello storico tra i crudeliter facta del secondo princeps [6].

I due principali studi moderni sulla vicenda di Cremuzio Cordo, quello di Gaetano Mario Columba (1901) e quello di Luciano Canfora (1993), liquidano abbastanza rapidamente la testimonianza dionea [7]; per quanto concerne la sezione del testo che apporta ulteriori informazioni sulle accuse contro l'imputato, in aggiunta alla lode di Bruto e Cassio: il primo le intende come una «parte, evidentemente, messa lì ad accrescer l'odiosità dell'imputazione» [8], il secondo scrive: «è evidente che qui siamo di fronte ad una amplificazione retorico-enfatica -riprendendo, sembrerebbe, I'idea del Columba-. Va da sé che non poteva costituire motivo o base di accusa il "non aver detto nulla di male su Cesare e Augusto ma non averli stralodati"! Questo già squalifica la notizia, per lo meno nella forma in cui la dà il tardo riassunto.

[4] Tac. Ann. IV, 34, 1-2: Cornelio Cosso Asinio Agrippa consulibus Cremutius Cordus postulatur novo ac tunc primum audito crimine, quod editis annalibus laudatoque M. Bruto C. Cassium Romanorum ultimum dixisset. Accusabant Satrius Secundus et Pinarius Natta, Seiani clientes. Trad. it.: «Sotto il consolato di Cornelio Cosso e Asinio Agrippa, Cremuzio Cordo fu accusato di un crimine nuovo e sentito allora per la prima volta, cioè per il fatto che pubblicata la sua opera annalistica e lodato M. Bruto aveva apostrofato C. Cassio come ultimo dei Romani. Ad accusarlo erano Satrio Secondo e Pinario Natta, clienti di Seiano».

[5] Il quale, per alcuni autori, non si sarebbe mai concluso con la condanna dell'imputato: vd. in proposito MARSH 1931, p. 290-293; Rogers 1935, p. 86-87; POSANI 1941, p. 1-13.; MARINo 2013, p. 47.

[6] Suet. Tib. 61: Singillatim crudeliter facta eius exequi longum est; genera, uelut exemplaria saeuitiae, enumerare sat erit.(...) Obiectum est poetae, quod in tragoedia Agamemnonem probris lacessisset; obiectum et historico, quod Brutum Cassiumque ultimos Romanorum dixisset (...). Trad. it.: «Sarebbe lungo descrivere dettagliatamente le sue azioni crudeli; sarà sufficiente enumerarne le categorie, come esempi di crudeltà. (...) A un poeta fu recriminato di aver infamato Agamennone in una tragedia; fu accusato anche uno storico, per il fatto che aveva apostrofato Bruto e Cassio come "gli ultimi dei Romani" (...)».

[7] Si aggiunga, inoltre, l'affermazione di Posani 1941, p. 1-2: «Le versioni di Tacito e Seneca presentano qualche divergenza, più apparente che reale del resto, mentre quelle di Svetonio e Dione Cassio sono troppo brevi e superficiali in confronto con le altre due, per poter servire di conferma all'una piuttosto che all'altra».

[8] Columba 1901, p. 22-23.

[9] CANFORA 1993, p. 226.

[10] Qualche accenno in MARINo 2013, p. 48-49.

[11] CANFORA 1993, p. 246

[12] Ibid. p. 221.
Altrettanto inconsistente appare la seconda imputazione: "di aver criticato il Senato e il popolo" (...) [9]».

Nondimeno, sembra discutibile che la testimonianza dionea, per quanto ripresa dal riassunto recenziore di Xifilino, aggiunga, senza alcuno scopo esplicativo, talune indicazioni sul caso in oggetto, con valore cioè meramente esornativo e retorico, senza piuttosto voler dare maggiore chiarezza al fatto storico [10].

Stante I'elogio dei cesaricidi di Cremuzio Cordo come capo d'imputazione condiviso, la critica è approdata a diverse soluzioni interpretative: il Columba fa riferimento alla lex Pedia de interfectoribus Caesaris, varata nel 43 a.C., la cui applicazione a questo caso sarebbe messa in atto con una differente pertinenza rispetto alla fattispecie criminosa originariamente contemplata dalla lex; il richiamo alla Lex Pedia, in quanto Ottaviano promotore, è presente anche in Canfora [11], il quale, parlando di reato di opinione [12], vi aggiunge anche la questione della licenziosità degli Annali di Cremuzio nei confronti di Augusto (Suet. Aug. 35) [13] e la necessità da parte degli accusatori di agire sulla base di un'esegesi estensiva della lex de maiestate [14]. Sulla stessa linea si pongono anche gli studi successivi condotti da S. $\mathrm{H}$. Rutledge, nel 2001 [15], e da S. Wilkinson, nel 2012 [16]; al contrario, divergono in maniera fondamentale dalla

\section{[13] Ibid. p. 230.}

[14] Ibid. p. 227.

[15] RutLeDGe 2001, p. 96, ha interpretato il capo d'accusa de maiestate come motivato dall'elogio di Bruto e Cassio (ancora, la sola motivazione fornita esplicitamente da Tacito in Ann. IV, 34), in quanto esso sarebbe contravvenuto al divieto promulgato da Augusto di fare menzione dei cesaricidi, dopo che peraltro lo stesso princeps ne aveva proibito l'esibizione delle imagines; Cfr. Tac. Ann. III, 76, 2: Viginti clarissimarum familiarum imagines antelatae sunt, Manlii, Quinctii aliaque eiusdem nobilitatis nomina. Sed praefulgebant Cassius atque Brutus eo ipso quod effigies eorum non visebantur. Trad. it.: «Davanti erano trasportati i ritratti di venti famiglie illustrissime, i Manlii, i Quinzii ed altri nomi della stessa nobiltà. Ma Cassio e Bruto splendevano sopra tutti, proprio per il fatto che non si vedevano le loro immagini».

[16] Per Wilkinson 2012, p. 41-42, l'indagine sul caso di Cremuzio Cordo deve essere condotta assumendo una prospettiva ideologica: l'imputato, avendo celebrato gli eroi "mitici" della res publica nella sua opera storica, non si presentava soltanto come un semplice nostalgico della genuina identità di uomo romano, ma definiva una categoria di "repubblicano" dalla quale Tiberio e i suoi rimanevano necessariamente esclusi; era, in ultima analisi, un oppositore del regime vigente, un potenziale sovversivo: «Under Tiberius, Cordus faced prosecution for a 'Republican' history. Whether Cordus was attacked for his past works, or whether he continued writing 'subversive' tracts under Tiberius, we do not know. Augustus presented his regime as an extension of the Republic and allowed praise of Brutus and Cassius and even Cato. One might have expected that Tiberius' insistence on following Augustan form and his emphasis on maintaining Republican tradition would guarantee continuity and Cordus' safety. But Cordus is an important marker for the opposition. He is the first man in Tacitus who attests to the end of the Republic and is thus a Republican martyr». 
communis opinio le riflessioni di M. R. McHugh (2004) [17] e di R. Marino (2013) [18], che riconoscono al testo dello storico bitinico una qualche rilevanza nell'analisi del fatto storico.

Con il presente studio ci si propone, dunque, di approfondire l'analisi delle versioni tràdite da Cassio Dione nella sua Storia Romana sul caso di Cremuzio Cordo (LVIII, 24, 2-4), proseguendo nella medesima direzione di quegli studiosi che hanno tentato di valorizzarne la testimonianza.

In primo luogo, ci si può interrogare su che cosa volesse esattamente esprimere annoverando tra le accuse contro

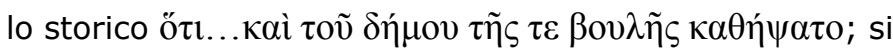
può -e si deve- pensare ad un'aggressione verbale [19], non fattuale [20], contro il Popolo e il Senato: peraltro il verbo $\kappa \alpha \theta \alpha ́ \pi \tau \omega$, componendosi di una preposizione, qui a reggenza genitivale [21], indicante un moto perlopiù ostile verso qualcuno o qualcosa, e di un lemma verbale che ha in sé I'idea dell'attacco, anche in senso fisico, e militare (ad esempio in Polibio [22]), rende bene l'immagine di un discorso dai toni accesi e risoluti contro il Popolo romano, cioè contro il solo istituto, insieme ai magistrati, tutelato dalla lex de maiestate in età repubblicana [23], che sarà poi affiancato dal Senato, dal princeps e dalla sua domus divina, qualora fossero lesi anche soltanto verbalmente [24]. In questo senso, la testimonianza dionea acquista significato in direzione di una sorta di perifrasi della sostanza del reato de maiestate, specificando pertanto il fatto che il processo contro Cremuzio Cordo fosse fondato sul reato di laesa maiestas, ora attributo, appunto, del Popolo di Roma e del suo Senato [25].

[17] McHugH 2004, p. 402 sottolinea che «Dio is the only one of the sources to say that Cremutius Cordus had not praised Caesar and Augustus enough. (...) Dio's statement indicates that the issue was one of control of speech rather than suppression of speech».

[18] Per MARINo 2013, p. 49, la testimonianza dionea «non rimane fuori dai circuiti di informazione sin qui analizzati, ma amplia la prospettiva alla luce della resistenza che il mito dei cesaricidi aveva registrato sino ai tempi dello storico dei Severi».

[19] Oltre che ai fatti, anche le parole iniziarono ad essere materiale d'accusa per il processo de maiestate in una seconda fase di sviluppo del reato: per la veridicità di quanto afferma Cremuzio Cordo in Tac. Ann. IV, 34, 2, vd. Manfredini 1979, p. 247-264. Per lo sviluppo del crimen maiestatis durante primi anni del principato vd. BAUMAN 1970, p. 246-265; Levick 1999, p. 151-159.

[20] Ma, non a torto, Borgo 2012, p. 41, scrive: «(...) il dissenso, una volta messo per iscritto, prende forma e concretezza e va combattuto in quanto pericolo fattuale».

[21] Per la voce verbale composta, costruita con il genitivo dipendente dalla preposizione, vd. Hdt. VI, 69; Th. VI, 16; Xen. Hell. I, 7, 4; Plat. Cri. 52a, 6.

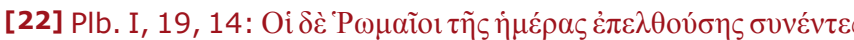

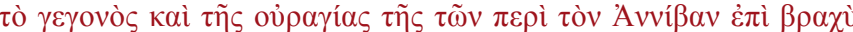

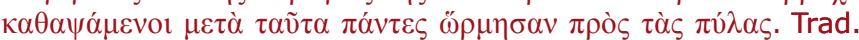
it.: «Il giorno seguente i Romani dopo aver appreso l'accaduto
Cicerone fornisce una chiara definizione della fattispecie criminosa abbracciata dal crimen maiestatis tra la fine del II e il I secolo a.C. [26]: Maiestatem minuere est de dignitate aut amplitudine aut potestate populi aut eorum, quibus populus potestatem dedit, aliquid derogare [27]. In essa compaiono due concetti chiave per la comprensione del significato del termine maiestas in età repubblicana: la detrazione della dignitas, della potestas e insieme dell'amplitudo, sia che si concretizzasse a danno del Popolo di Roma sia dei suoi magistrati, comportava la consumazione di un crimine, non solo nei riguardi di questi ultimi, ai quali era affidato il potere, ma soprattutto nei confronti del Populus Romanus, il solo corpo politico cui fosse lecito delegare il potere attraverso I'honor, ovvero la magistratura; perciò, I'oltraggio e la sottrazione di dignitas, potestas e amplitudo, perpetrata ai danni di un magistrato, implicava necessariamente anche l'offesa dello stesso Populus, da cui ogni potere, direttamente o indirettamente, traeva origine [28]. Il fatto che Augusto abbia modificato il raggio d'applicazione della legge, estendendolo alla sua stessa persona e alla domus divina, è ben comprensibile alla luce del fatto che il princeps, investito della sacrosanctitas in forza della sua tribunicia potestas [29], era di fatto anche il magistrato più importante della nuova respublica restituta, grazie al valore aggiunto del grado superiore di auctoritas esercitabile [30] accanto alla potestas magistratuale, divenendo, in questo senso, davvero princeps.

Del resto, si muove in questa direzione anche il significato precipuo del verbo impiegato, $\kappa \alpha \theta \alpha ́ \pi \tau \omega$ [31], attestato già

e attaccato per un po' di tempo la retroguardia dell'esercito di Annibale, si diressero tutti insieme verso le porte della città».

[23] BAUMAN 1970, p. 8-15.

[24] Ibid. p. 288.

[25] MACKIE 1992, p. 88-91. Cfr. Tac. Ann. I, 72.

[26] Cic. Invent. II, 53: «Sminuire la maiestas consiste nel sottrarre qualcosa dalla dignità, dall'autorità o dal potere del popolo o di coloro ai quali il popolo ha affidato il potere».

[27] Cfr. anche Cic. Pis. 49-50; Fam. III, 11, 2.

[28] BAUMAN 1970, p. 88-90.

[29] Aug. RGDA, 10, 1: Nom[en me]um [sena]tus c[onsulto inc]lusum est in saliare carmen, et sacrosanctu[s in perp] $t<u>m$ [ut essem et, q]uoad viverem, tribunicia potestate mihi e[sset, per lege]m st[atutum est. Trad. it.: «Il mio nome per decreto del senato è stato inserito nel carmen saliare, ed è stato stabilito per mezzo della legge che io fossi inviolabile per l'eternità, e che, fino alla mia morte, fossi investito della tribunicia potestas».

[30] Ibid. 34, 3: Post id temp[us a]uctoritate [omnibus praestiti, potest]atis au[tem n]ihilo ampliu[s habu]i quam cet[eri, qui m]ihi quoque in ma[gis]tra[t]u conlegae f[uerunt]. Trad. it.: «In seguito a ciò fui superiore a tutti per auctoritas ma non ebbi affatto un maggior grado di potestas rispetto agli altri che furono colleghi insieme a me in ogni magistratura».

[31] Cfr. SAILOR 2008, p. 282, n. 70. 
a partire dai testi omerici in diverse occorrenze [32]. La voce verbale, di cui è possibile evidenziare la grande vitalità d'impiego, ricorre con la stessa sfumatura di significato (manifestazione verbale di ostilità/riprovazione) in opere di vario genere letterario [33]; per quanto concerne l'opera di Cassio Dione, essa è presente anche in ulteriori due luoghi della sua opera storica (Dio XLVI, 1, 1 [34] e Dio LXXV, 8, 2 [35]), adoperata in riferimento ad un discorso orale pubblico, come può essere il caso di un dibattito tenuto in senato, secondo un impiego che ne rispetta I'accezione originaria.

Ad avvalorare la possibilità che I'opera scritta non sia stata la sola causa che portò al processo dello storico, viene in aiuto proprio uno dei testimoni decisivi, Seneca, che in Cons. Marc. 22, 4 riferisce dell'ira di Seiano infiammata dalle parole dirette contro di lui da Cremuzio Cordo, dando l'impressione di voler mettere in correlazione la citazione riportata all'accusa de maiestate. Scrive così I'autore della Consolatio: (...) Decernebatur illi statua (scil.: Seiano) in Pompei theatro ponenda, quod exustum Caesar reficiebat: exclamavit Cordus tunc vere theatrum perire [36]. Sembrerebbe appropriato riferire la menzione riportata da Seneca non tanto all'opera annalistica composta dallo storico imputato, quanto piuttosto a possibili interventi tenuti verosimilmente in senato da Cremuzio Cordo. Un buon momento per un intervento personale di Cremuzio Cordo potrebbe essere stato appunto I'occasione [37] in cui venne approvata I'erezione nel Teatro di Pompeo di una statua onoraria

[32] Ancor più probante il fatto che quest'ultimo nei poemi epici costituisca, nella maggior parte dei casi, un tipico caso di nesso formulare in unione con il lemma ع́ $\pi$ o $\zeta$ opportunamente declinato (Cfr. Hom. II. XV, 121; XVI, 421; Od. II, 240; XVIII, 415; XX, 22), la cui radice originaria designa l'azione del parlare attraverso l'emissione della voce (laddove, si noti, proprio il sostantivo latino vox conserva la medesima radice protoindoeuropea ${ }^{*} w e k w$ - presente in $\varepsilon \pi^{\prime} \zeta_{\zeta}$, per cui vd. ora ERnout \& Meillet 1951, p. 1332-1330; Chantraine 1970, p. 362), ovvero la performance orale.

[33] Oltre ai luoghi già citati per Omero, si segnalano: Hes. Op. 332; Hdt. VI, 69; Thuc. VI, 16; VI, 82. Xen. Hell. I, 7, 4; Plat. Cri. 52a, 6; Plut. Ages. 8, 13; Galb. 26, 1.

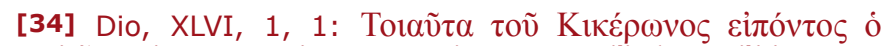

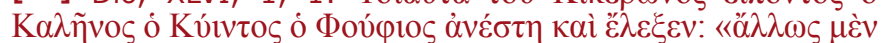

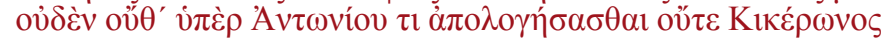

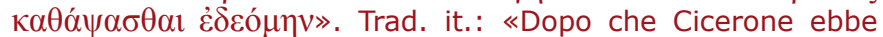
detto queste parole Quinto Fufio Caleno si alzò in piedi e disse: "non dirò nient'altro nemmeno in difesa di Antonio né ho bisogno di attaccare Cicerone"».

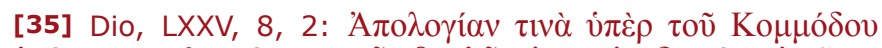

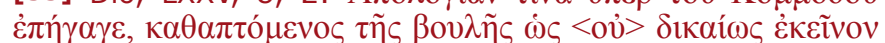

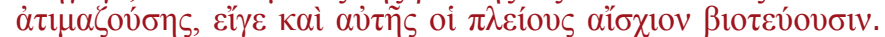
Trad. it.: «Pronunciò un discorso in difesa di Commodo, attaccando il senato per il fatto che lo aveva disonorato ingiustamente, nonostante la maggior parte di esso vivesse in modo ancor più vergognoso».

[36] Per l'intero passo accompagnato dalla traduzione vd. supra nota n. 3.

[37] Sen. Marc. 22, 4 per Seiano, intorno agli anni 21-22 d.C., allorché l'edificio veniva restaurato dopo un incendio [38], circostanza nella quale avrebbe potuto deplorare con troppa franchezza (unum aut alterum liberius dictum) la connivenza del popolo e soprattutto del senato all'ascesa al potere di Seiano (quod tacitus ferre non potuerat Seianum in cervices nostras ne inponi quidem sed escendere), ma anche «parlare di Augusto e di Cesare senza dirne male, ma nemmeno lodandoli troppo». Infatti, proprio in quell'occasione, il senatore avrebbe potuto richiamare alla memoria di tutti i patres il precedente restauro di Ottaviano, risalente al 32 a.C., anno di cui certamente Cremuzio Cordo deve aver parlato nella sua opera $\pi \varepsilon \rho i$

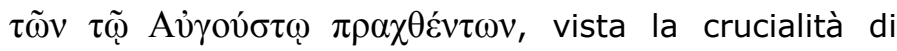
quel decennio per la politica del futuro Augusto, tanto più perché si trattava di un intervento pubblico di cui lo stesso princeps si vantò nel suo testamento politico [39]. Del primo rimaneggiamento, che coinvolse inoltre la Curia di Pompeo del cui complesso faceva parte il Teatro, dà notizia anche Svetonio, nella cui opera si può leggere una sorta di elogio rivolto a quest'azione promossa da Ottaviano, il quale avrebbe fatto spostare la statua di Pompeo dalla Curia al portico antistante al Teatro, dal momento che, sebbene la Curia in cui fu assassinato Cesare fosse stata bollata come locus sceleratus e quindi murata [40] e riutilizzata più tardi come latrina [41], comunque l'onore di Pompeo quale exemplar per i cives ne usciva salvo, e per di più riconosciuto proprio dal figlio di Cesare [42]. Ma bisogna ancora una volta ritornare a

[38] Cfr. Tac. Ann. III, 72, 2-4.

[39] Aug. RGDA, 20, 1: Capitolium et Pompeium theatrum utrumque opus impensa grandi refeci sine ulla inscriptione nominis mei. Trad. it.: «Ho fatto restaurare sia il Campidoglio, sia il teatro di Pompeo ed entrambi spendendo molto denaro senza farvi iscrivere sopra il mio nome». Vd. inoltre Schettino 2015, p. 353-372.

[40] Suet. Iul. 88: Curiam, in qua occisus est, obstrui placuit Idusque Martias Parricidium nominari, ac ne umquam eo die senatus ageretur. Trad. it.: «Si decise di murare la Curia, nella quale fu ucciso, di denominare le Idi di Marzo "Parricidio", e che mai il senato si riunisse durante quel giorno».

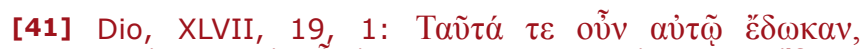

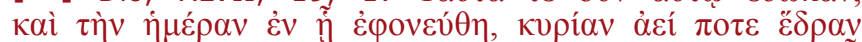

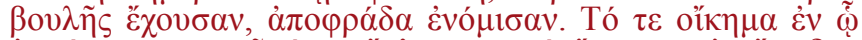

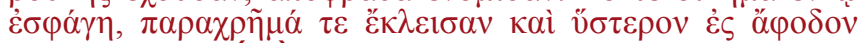
$\mu \varepsilon \tau \varepsilon \sigma \kappa \varepsilon v ́ \alpha \sigma \alpha \nu$ (...). Trad. it.: «Dunque questo fu quanto gli venne decretato, e il giorno nel quale fu assassinato, durante il quale il senato si era sempre riunito, venne detto nefasto. L'edificio in cui fu ucciso, venne subito chiuso e in seguito venne trasformato in una latrina $(. .)$.$» .$

[42] Suet. Aug. 31: Pompei quoque statuam contra theatri eius regiam marmoreo Iano superposuit translatam e curia, in qua $C$. Caesar fuerat occisus. Trad. it.: «Fece anche collocare la statua di Pompeo trasportata dalla Curia, in cui era stato ucciso Cesare, di fronte alla scena del teatro di Pompeo su un arco di marmo». 
leggere Seneca: Quid ergo? Non rumperetur supra cineres Cn. Pompei constitui Seianum et in monumentis maximi imperatoris consecrari perfidum militem? Consignatur subscriptio, et acerrimi canes, quos ille, ut sibi uni mansuetos, omnibus feros haberet, sanguine humano pascebat, circumlatrare hominem etiam illo in peri<culo inperturb>atum incipiunt. Quid faceret? [43] Proprio da questo passo senecano risulta chiaro come Seiano, ora «innalzato sopra alle ceneri di Pompeo», sarebbe andato a sostituirsi e ad oscurare l'imago di quello che era ormai divenuto un simbolo della Repubblica, un'ombra con cui dovette costantemente confrontarsi anche Augusto in più occasioni. È allora possibile che lo spirito di libertà con cui Cremuzio si era espresso nella sua opera, non avesse esitato a metterlo in pratica di fronte alla stessa Roma: la lettura delle testimonianze antiche spinge a pensare che Cremuzio Cordo, diventato a sua volta un simbolo di libertas già a pochi anni di distanza dal suo "martirio", non solo aveva osato scrivere un'opera di storia contemporanea in tutta franchezza, ma neppure a parole dimostrava di essere di meno un homo ingenio animo manu liber (Sen. Marc. 1, 3). Nello stesso senso potrebbe dunque essere letta una delle espressioni dionee prese

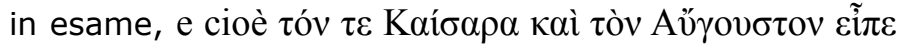

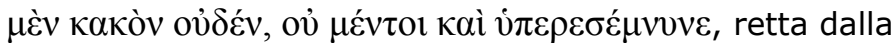
stessa preposizione epesegetico-causale da cui dipende I'intero periodo. La sinteticità dell'espressione ne oscura infatti la precisa accezione: si potrebbe nuovamente fare riferimento a determinati luoghi dell'opera annalistica di Cremuzio, ma, allo stesso tempo, si potrebbe pensare anche a discorsi tenuti in pubblico, gli stessi nei quali egli avrebbe potuto infamare il nome del Senato e del Popolo romano, attaccandolo per il suo servilismo nei confronti di Seiano - il potente prefetto contro il quale si conosce per certo I'odio nutrito da Cremuzio Cordo [44]. Anche

[43] Sen. Marc. 22, 5: «E allora? Non ci si altererebbe per un Seiano innalzato sulle ceneri di Pompeo e per un soldato infido consacrato nei luoghi della memoria di un grandissimo generale? Si presenta l'atto di accusa, e quei cani rabbiosi, che egli nutriva con sangue umano, affinché fossero docili con lui solo, e con tutti feroci, iniziarono ad abbaiare intorno a quell'uomo anche in quell'occasione imperturbabile. Che fare?».

[44] Così lascerebbe intendere anche Tacito, secondo lo studio di WISSE 2013 , p. 316: «In summary, Cremutius' trial occurs in a part of the Annals where the focus has increasingly been on depressing domestic affairs, and it is preceded by a digression interpreting such historiographical focus as an ominous sign of imperial times. Tacitus has presented a tightening plot, by depicting parallel developments of repression through maiestas trials and of senatorial impotence and adulation, recently compounded by the growth of Sejanus power; and he has emphasised these developments by means of a number of 'landmarks'». A questo tema accenna concisamente anche MARINO 2013, p. 49, che, malgrado la diversa finalità d'interpretazione, scrive: «nella parte relativa alle accuse appare il più critico e, insieme, il più ironico, nello in questo senso, dunque, Cremuzio Cordo fu accusato e ricacciato nella dimenticanza ob duas res pulcherrimas, eloquentiam ac libertatem [45].

Svetonio, in Aug. 35, dimostra di conoscere I'opera annalistica di Cremuzio, e trasmette una notizia attinta ad essa riguardante il principato augusteo (lectio del 18 a.C.): Senatorum affluentem numerum deformi et incondita turba (erant enim super mille, et quidam indignissimi et post necem Caesaris per gratiam et premium adlecti, quos orcivos vulgus vocabat) ad modum pristinum et splendorem redegit duabus lectionibus: prima ipsorum arbitratu, quo vir virum legit, secunda suo et Agrippae; quo tempore existimatur lorica sub veste munitus ferroque cinctus praesedisse, decem valentissimis senatorii ordinis amicis sellam suam circumstantibus. Cordus Cremutius scribit ne admissum quidem tunc quemquem senatorum nisi solum et praetemptato sinu [46]. In questo passo della biografia svetoniana, la lectio senatus indetta da Augusto, coadiuvato da Agrippa, trascina inevitabilmente con sé, accanto alla menzione del princeps, anche quella di Cesare, a causa del quale il Senato era divenuto un ammasso traboccante di orcivos, e un ritratto canzonatore di quest'ultimo: purtroppo però, non si ha la certezza che questa associazione fosse contenuta già nell'opera di Cremuzio, dal momento che Svetonio introduce il nome del suo auctor soltanto dopo un punto fermo, e all'inizio di un nuovo periodo che introduce la trasmissione di una nuova informazione, pur proseguendo nella descrizione della stessa lectio senatus del 18 a.C.

Una conferma del fatto che Cremuzio abbia, almeno, parlato in questi termini della coppia Augusto-Cesare si potrebbe ricavare dalla lettura del contenuto dell'apologia messa in bocca da Tacito a Cremuzio Cordo in Ann. IV, 34, 2-35,4, ponendo I'accento sul fatto che l'imputato

sforzo di fare emergere l'asimmetria della pena rispetto alla colpa, anche attraverso tecniche allusive - il servilismo del senato e del popolo - difetto da cui Cordo è esente».

[45] Sen. Marc. 1,4: Magnum mehercules detrimentum respublica ceperat, si illum ob duas res pulcherrimas in oblivionem coniectum, eloquentiam et libertatem, non eruisses (...). Trad. it.: «Lo stato avrebbe subito un grande danno, se tu non lo avessi disseppellito, ormai condannato alla dimenticanza per due cose bellissime, I'eloquenza e la libertà!».

[46] Suet. Aug. 35: «Ricondusse con due lectiones il numero traboccante dei senatori, folla scomposta e informe, all'antico splendore (erano infatti oltre mille, alcuni assolutamente indegni e accorpati dopo la morte di Cesare grazie a favori e denaro, e il popolo li chiamava "senatori d'oltretomba"): la prima secondo la loro stessa volontà, per cui ognuno sceglie I'altro, la seconda secondo la sua e quella di Agrippa; in quel tempo si credeva che presiedesse in senato munito di lorica sotto la toga e cinto di spada, circondando la sua sella curule dieci suoi robustissimi amici dell'ordine senatorio. Cremuzio Cordo scrive che allora nessuno dei senatori era fatto entrare se non da solo e dopo essere stato perquisito». 
nella ricostruzione tacitiana avrebbe annoverato tra precedenti il nome di Augusto e di Cesare in qualità di comparanda autorevoli; in particolare in Ann. IV, 34, 3-5, egli avrebbe addotto a riprova della tolleranza (ovvero della saggezza politica) usata precedentemente in contesti simili l'atteggiamento di Augusto nei confronti di Tito Livio [47], delle lettere di Antonio e dei discorsi di Bruto [48], quello di Cesare nei riguardi di Cicerone [49] e di Catullo, e la disposizione di entrambi verso le offese poetiche di Furio Bibaculo, commentando ambiguamente nella chiusa: haud facile dixerim moderatione magis an sapientia [50]. Ammettendo, secondo la testimonianza tacitiana, che Cremuzio Cordo abbia realmente pronunciato in termini simili un'apologia in senato prima della sua condanna [51], e considerato il fatto che Cassio Dione, così come lo stesso Tacito [52], ha fatto uso nella redazione della sua opera storica di documenti ufficiali, quali sono gli acta senatus [53], si può ipotizzare che entrambi abbiano attinto ad essi anche nel reperimento di informazioni sul processo di Cremuzio Cordo. Perciò con

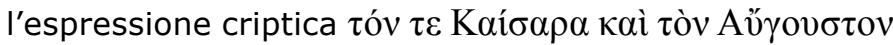

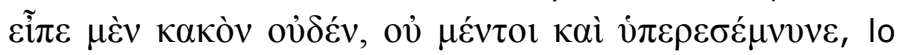
storico bitinico potrebbe aver sintetizzato quella parte del testo degli acta che registrava il discorso di difesa pronunciato da Cremuzio nel 25 d.C. il quale, stando a Tacito che si diffonde più ampiamente sulla questione, avrebbe chiamato in causa Cesare e Augusto come

[47] Tac. Ann. IV, 34, 3-4: Titus Livius, eloquentiae ac fidei praeclarus in primis, Cn. Pompeium tantis laudibus tulit, ut Pompeianum eum Augustus appellaret; neque id amicitiae eorum effecit. Scipionem, Afrianum, hunc ipsum Cassium, hunc Brutum nusquam latrones ac parricidas, quae nunc vocabula imponuntur, saepe ut insigni<s> viros nominat Trad. it.: «Tito Livio, tra i più distinti per eloquenza e per fedeltà storica, lodò Pompeo con tanti elogi che Augusto lo denominò "pompeiano"; ma ciò non intaccò la loro amicizia. Scipione, Afranio, questo stesso Cassio, questo Bruto non II chiama in nessun luogo banditi o parricidi, termini che ora sono imposti, ma spesso li menziona quali uomini illustri».

[48] Tac. Ann. IV, 34, 5: Antonii epistulae, Bruti contiones falsa quidem in Augustum probra, set multa cum acerbitate habent (...). Trad. it.: «Le lettere di Antonio, i discorsi di Bruto contengono certo infamanti falsità contro Augusto, ma condite da grande durezza».

[49] Tac. Ann. IV, 34, 4: Marci Ciceronis libro, quo Catonem caelo aequavit, quid aliud dictator Caesar quam rescripta oratione, velut apud iudices, respondit? Trad. it.: «Al libro di Marco Cicerone in cui questi innalza Catone al cielo, in che altro modo il dittatore Cesare rispose se non con un discorso di replica messo per iscritto, come se si fosse davanti ai giudici?».

[50] Tac. Ann. IV 34, 5: (...) carmina Bibaculi et Catulli referta contumeliis Caesarum leguntur: sed ipse divus Iulius, ipse divus Augustus et tulere ista et reliquere, haud facile dixerim, moderatione magis an sapientia. Namque spreta exolescunt: si irascare, adgnita videntur. Trad. it.: «(...) i componimenti di Bibaculo e di Catullo sono pieni di offese contro i Cesari nondimeno lo stesso divo Giulio, lo stesso divo Augusto sopportarono ciò e lasciarono correre, non saprei dire facilmente se più per moderazione o per saggezza. Infatti ciò che viene precedenti autorevoli per il suo caso, sebbene non dandone un giudizio del tutto favorevole considerata I'ambiguità dell'espressione. E non si può escludere che queste stesse parole di autodifesa gli fossero state ritorte contro immediatamente, giacché il processo venne abbandonato in itinere dall'imputato [54]. Tale ipotesi dimostrerebbe che l'accordo tra Tacito, considerata la fonte principale, e Cassio Dione, stimato in questo contesto testimone di secondo piano, è più stringente di quanto non sembri a prima vista, contribuendo così a incrementare la validità sul piano storico della testimonianza dionea.

L'animus liber di Cremuzio doveva certamente trasparire nella sua opera storica. Seneca Padre, ad esempio, riferisce di una lode, presumibilmente contenuta nei suoi Annales, che lo storico, in prima persona, aveva tributato a Cicerone dopo la sua morte [55], personaggio sul quale si era soffermato anche in altri luoghi della sua opera [56]. La simpatia per la factio repubblicana doveva essere presente anche altrove: la stessa lode dei cesaricidi probabilmente fu fatta propria da Cremuzio Cordo [57] o attribuitagli, con secondi fini, dai suoi accusatori; essa è, in effetti, da scomporsi sulla base di Tacito, in una climax ascendente che prevede la lode di Bruto e I'apostrofe di Cassio come ultimus Romanorum; che quest'epiteto elogiativo fosse stato attribuito da Bruto a Cassio al momento della sua morte, è tramandato da

disprezzato ricade nella dimenticanza: se ci si abbandona alla collera sembra essere vero».

[51] Per la veridicità del discorso di Cremuzio Cordo in Tacito: CANFORA 1993, p. 236. Cfr. anche Posani 1941, p. 1-13; contra: ColumBA 1901, p. 18-19; SYME 1958, p. 337-338; Meier 2003, p. 107-110; McHugh 2004, p. 391-392.

[52] SYME 1958, p. 187-188; Gonzalez 2002, p. 52.

[53] Vd. LETTA 2003. Per l'impiego degli acta nella narrazione della vicenda di Cremuzio, vd. CANFOrA 1993, p. 242-244 che però propende per una derivazione diversa dagli acta senatus.

[54] Sen. Marc. 22, 7-8: Accusatores auctore Seiano adeunt consulum tribunalia, queruntur mori Cordum, ut interpellarent quod coegerant: adeo illis Cordus videbatur effugere. Magna res erat in questione, an mortis <ius> rei perderent; dum deliberatur, dum accusatores iterum adeunt, ille se absolverat. Trad. it.: «Incitati da Seiano gli accusatori adivano i tribunali dei consoli, lamentando che Cordo morisse, perché impedissero ciò che essi lo costringevano a fare: a tal punto Cordo sembrava loro sfuggire via. Qualcosa di grande era in discussione, se cioè gli imputati perdessero il diritto di morire; mentre si discuteva, mentre gli accusatori tornavano ancora, egli si era assolto da sé».

[55] Sen. Suas. VI, 23: Non est operae pretium referre redditam Ciceroni laudationem, nihil enim in ea Cicerone dignum est ac ne hoc quidem quod paene maxime tolerabile est (...). Trad. it.: «Non vale la pena riferire la "laudatio" tributata da Cremuzio a Cicerone; infatti quasi nulla in essa è degno di Cicerone e nemmeno questo passo, che è il più tollerabile (...)».

[56] Sen. Suas. VI, 19.

[57] Così già NiPPERDEY 1851, p. 127. 
Appiano [59] e Plutarco [58], per i quali è stata peraltro proposta una derivazione diretta della notizia dall'opera di Cremuzio [60].

Se dunque I'opera storica ha, in un certo senso, riflesso i sentimenti repubblicani di Cremuzio [61], è allora naturale che Seiano e i suoi clientes si siano rivolti ad essa per mettere le mani su una prova concreta, necessaria per poter presentare un atto di accusa contro lo storico e per instaurare un processo accusatorio contro di lui; per questo motivo si può pensare che I'opera storica sia esplicitamente indicata dalle totalità delle fonti come la sola causa del processo contro Cremuzio, in quanto capo di imputazione presente nel libellus sottoscritto dagli accusatori [62]: a questo proposito Cassio Dione, spesso attento agli aspetti giuridico-istituzionali, sembra voler puntualizzare da una parte il capo d'imputazione e dall'altra l'insieme delle accuse, utilizzando dapprima

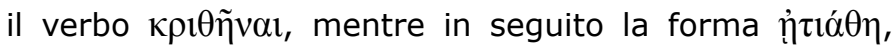

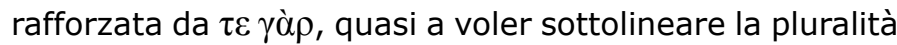
delle incriminazioni [63].

La testimonianza dionea potrebbe suggerire, data la specificità del termine impiegato ( $\kappa \alpha \theta \alpha \dot{\pi} \tau \omega)$, che le parole di Cremuzio Cordo imputate di laesa maiestas, almeno limitatamente ai punti qui presi in esame, possano essere state tanto pronunciate quanto messe per iscritto [64], ipotizzando che Cremuzio, dato il suo ruolo di senatore, sia potuto intervenire nel consesso dei patres durante una discussione tenuta nella Curia, e che in essa abbia potuto spendere qualche parola di disapprovazione nei confronti

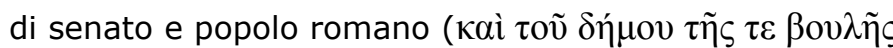
$\kappa \alpha \theta \eta ́ \psi \alpha \tau o)$ e si sia espresso su Cesare e Ottaviano,

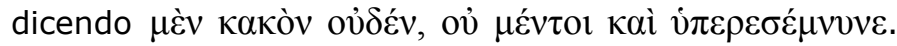
Si è dunque rilevata la possibilità che le accuse contro Cremuzio siano state ricavate tanto dall'opera storica, la cui esistenza materiale era indispensabile per l'apertura del processo, quanto da verba realmente pronunciati dallo stesso imputato, come potrebbe sottintendere l'analisi delle forme verbali impiegate [65]. Bisogna ora volgere

[58] App. IV, 15, 114.

[59] Plut. Brut. 44.

[60] Così già Hinz 1891, p. 76-79; cfr. GotTer 1996, p. 20 e nota n. 45.

[61] Similmente, per esempio, Seneca fa parlare Cremuzio in Marc. 26, 3: (...) ego nihil in me cuiquam permisi et cibo prohibitus ostendi tam magno me quam vivebam animo scripsisse. Trad. it.: «Io a nessuno ho permesso di infierire contro di me e privandomi del cibo ho mostrato di aver scritto con la stessa grandezza d'animo con cui vivevo».

[62] Sembra infatti tecnico il consignatur subscriptio di Sen. Marc. 22, 5

[63] Il passo si può confrontare in parallelo con le forme postulatur e accusabant presenti in Tac. Ann. IV, 34, 1.

[64] Cfr. KLINGER 1958, p. 197: «Die Anklage betraf also Worte in dem Geschichtswerk, offenbar, weh sie darin versteckte,
I'attenzione alla criticità degli anni appena precedenti il processo.

A partire dal 20 d.C. I'influenza di Seiano continuava ad accrescersi sempre di più: in questo stesso anno gli vennero conferiti gli ornamenta praetoria; nel 22 d.C. la sua potenza veniva tradotta in immagine visibile a tutti attraverso l'erezione di una statua in suo onore; nel 23 d.C., sopraggiunge la morte di Druso [66] che, possibile successore al regno in seguito alla morte di germanico, si trovava in aperta rivalità con Seiano [67] il quale, otto anni più tardi e ormai defunto, fu accusato da Apicata di aver architettato il suo avvelenamento insieme a Livia, vedova di Druso e amante di Seiano [68]; nello stesso anno, venivano costruiti i Castra Praetoria sul colle del Viminale: il suo prestigio politico veniva corroborato dall'autorità militare. Narrando i fatti dell'anno 23 d.C. Tacito scrive: (...) cum repente turbare fortuna coepit, saevire ipse aut saevientibus vires praebere. Initium et causa penes Aelium Seianum cohortibus praetoriis praefectum, cuius de potentia supra memoravi: nunc originem, mores, et quo facinore dominationem raptum ierit expediam [69].

In definitiva, il 23 d.C., è I'anno che meglio può essere interpretato come il momento decisivo dell'ascesa al potere di Seiano. Solo due anni dopo, nel 25 d.C., Cremuzio sarebbe stato processato de maiestate: ma è ragionevole pensare, sulla base della preziosa testimonianza senecana, che almeno a partire dal 21-22 d.C. I'avversione del senatore nei riguardi della scalata al potere del prefetto fosse manifesta, verosimilmente attraverso il disappunto che Cremuzio in un clima simile a quello della decretazione della statua avrebbe potuto dichiarare sia attaccando direttamente Seiano, sia il senato e il popolo proni al volere del primo, come lo stesso Tiberio [70]. Tuttavia, era necessario impadronirsi di prove concrete: niente di meglio della vecchia opera storica, in cui tentare di identificare la trascrizione di quei sentimenti che Cremuzio aveva già espresso pubblicamente, ma che non

mittelbare Angriffe auf Cäsar und Augustus und damit auf die bestehende Ordnung des Prinzipats sah».

[65] L'allusione ai due ordini di motivi è in particolare nella

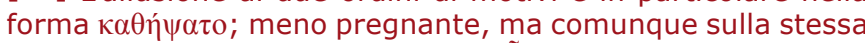
linea, si potrebbe porre la forma $\varepsilon \tilde{\pi} \pi \varepsilon$. Cfr. anche la discussione in HeNNig 1975, p. 55-63.

[66] Tac. Ann. IV, 7-8.

[67] Tac. Ann. IV, 3.

[68] Tac. Ann. IV, 10-11.

[69] Tac. Ann. IV 1 : «(...) quando d'un tratto la sorte iniziò a turbarsi, lui stesso a divenire crudele oppure a incoraggiare chi già lo era. Principio e causa Elio Seiano, prefetto delle coorti pretorie, della cui potenza ho già parlato: ora riferirò le origini, i costumi e con quale misfatto giunse ad impossessarsi del potere assoluto».

[70] Così puntualizza icasticamente Sen. Marc. 22, 4: ne imponi quidem, sed escendere. 
gli potevano essere imputati contro perché privi di un'attestazione tangibile, di una prova direttamente imputabile alla sua persona, come invece erano i suoi Annali.

In conclusione, in questa nota al libro LVII della Storia Romana di Cassio Dione si è voluto tentare di valorizzare le testimonianze dello storico bitinico, le quali si crede possano illuminare dettagli scarsamente chiari e di difficile comprensione. Nel testo preso in analisi, I'attendibilità di Cassio Dione riluce sia nella probabile conformità con il testo tacitiano, nella misura in cui entrambi gli storici abbiano tratto dalla stessa documentazione ufficiale degli acta senatus le notizie trasmesse, sia nella consonanza di punti chiave con le testimonianze senecane contenute nella Consolatio ad Marciam [71], di cui si è già ricordata I'importanza per un'interpretazione ragionata del caso di Cremuzio Cordo, in quanto fonte storica di prima mano.

[71] Per le varie ipotesi di datazione dell'opera (che comunque non dovrebbe essere stata composta a più di un venticinquennio dalla morte di Cremuzio Cordo), vd. la discussione in BeLLEMORE 1992.

Cary, Earnest \& Foster, Herbert Baldwin, 1924, Dio Cassius. Roman History, Volume VII: Books 56-60, Cambridge - London.

Casorati, Francesco \& De Salvo, Lietta, 2008, Gaio Svetonio Tranquillo. Vita dei Cesari, Roma.

Cornell, Timothy (ed.), 2013, The Fragments of the Roman Historians, Oxford.

Goodyear, Francis Richard David, 1981, The Annals of Tacitus. Volume II: Annals I,55-81 and Annals II, Cambridge.

Martin, Ronald \& Woodman, Anthony John, 1989, Tacitus, Annals. Book IV, Cambridge.

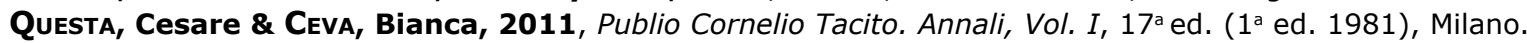

Scheid, John, 2007, Res gestae divi Augusti. Hauts faits du divin Auguste, Paris (Les Belles Lettres).

Sordi, Marta, Stroppa, Alessandro, Galimberti, Alessandro, 2014, Cassio Dione. Storia Romana, volume VI (Libri LVIILXIII), 6a ed. (1 $1^{\text {a }}$ ed. 1999), Milano.

Traina, Alfonso, 2010, Seneca. Le consolazioni, $11^{\text {a }}$ ed. (1 $1^{\text {a }}$ ed. 1987), Milano.

Woodman, Anthony John \& Martin, Ronald, 1996, Tacitus, Annals. Book III, Cambridge - New York.

Bauman, Richard Alexander, 1970, The crimen maiestatis in the Roman Republic and Augustan Principate, $2^{\mathrm{a}}$ ed. (1 $1^{\mathrm{a}}$ ed. 1967$)$, Johannesburg.

Bauman, Richard Alexander, 2004, Crime and Punishment in Ancient Rome, 2a ed. (1 $1^{\mathrm{a}}$ ed. 1996), London.

Bellemore, Jane, 1992, «The dating of Seneca's ad Marciam de consolatione», CQ XLII, 1, p. 219-234.

Borgo, Antonella, 2012, «Res nova et inusitata, supplicium de studiis sumi (Sen. contr. 10 praef. 5): a proposito dei roghi di libri a Roma», Paideia LXVII, p. 33-53.

Canfora, Luciano, 1993, Studi di storia della storiografia romana, Bari.

Chantraine, Pierre, 1970, Dictionnaire étymologique de la langue grecque II (E-K), Paris.

Columba, Gaetano Mario, 1901, «Il processo di Cremuzio Cordo», Atene e Roma XXXV, p. 1- 44.

Ernout, Alfred \& Meillet, Antoine, 1951, Dictionnaire étymologique de la langue latine, $3^{\mathrm{a}}$ ed. (1 $1^{\mathrm{a}}$ ed. 1932), Paris.

Gonzalez, Julian, 2002, Tacito y las fuentes documentales: SS. CC. de honoribus Germanici decernendis (Tabula Siariensis) et de Cneo Pisone Patre, Sevilla.

GotTeR, Ulrich, 1996, Der Diktator ist tot!: Politik in Rom zwischen den Iden des März und der Begründung des Zweiten Triumvirats, Stuttgart. 
Gowing, Alain Michael, 2009, «From the Annalists to the Annales: Latin Historiography before Tacitus», in Woodman, Anthony J. (ed.), The Cambridge Companion to Tacitus, Cambridge, p. 17-30.

Hennig, Dieter, 1975, Lucius Aelius Seianus. Untersuchungen zur Regierung des Tiberius, München.

Hinz, Christian H., 1891, Zur Beurteilung Appians und Plutarchs in der Darstellung der Ereignisse von der Ermordung Cäsars bis zum Tode des M. Brutus, Jena.

KLINGer, Friedrich, 1958, «Tacitus und die Geschichtsschreiber des 1. Jahrhunderts n. Chr.», MH XV, p. $194-206$.

LaVALLe, Domenica, 2012, «Il potere delle azioni dissidenti: Augusto e i suoi oppositori», Paideia LXVII, p. 187-202.

LetTA, Cesare, 2003, «Documenti d'archivio e iscrizioni nell'opera di Cassio Dione: un sondaggio nella narrazione fino ad Augusto», in Anna Maria Biraschi, Paolo Desideri, Sergio Roda, Giuseppe Zecchini (edd.), L'uso dei documenti nella storiografia antica, Napoli, p. 595-622.

Levick, Barbara, 1999, Tiberius the Politician, $3^{\text {a }}$ ed. (1 ${ }^{\text {a }}$ ed. 1976), London - New York.

MACKIE, Nicola, 1992, «Ovid and the Birth of Maiestas», in Anton Powell (ed.), Roman Poetry and Propaganda in the age of Augustus, Bristol, p. 83-97.

Manfredini, Arrigo, 1979, La diffamazione verbale nel diritto romano, Milano.

Marino, Rosalia, 2013, «Sul "processo" a Cremuzio Cordo», "O $\rho \mu o \varsigma$ 5, p. 44-55.

MARSH, Frank Burr, 1931, The Reign of Tiberius, Oxford.

MeIer, Mischa, 2003, «Das Ende des Cremutius Cordus und die Bedingungen für Historiographie in augusteischer und tiberischer Zeit», Tyche 18, p. 91-127.

McHugh, Mary, 2004, «Historiography and Freedom of Speech: the Case of Cremutius Cordus», in Ineke Sluiter \& Ralph Mark Rosen (ed.), Free speech in classical antiquity, Leiden - Boston, p. 391-408.

Migliario, Elvira, 2007, Retorica e storia. Una lettura delle Suasoriae di Seneca Padre, Bari.

Posani, Maria Rosa, 1941, «La veridicità storica di Tacito a proposito del processo di Cremuzio Cordo», Rendiconti della classe di scienze morali e storiche della Reale accademia d'Italia VII, 2, p. 1-13.

Rogers, Robert Samuel, 1935, Criminal Trials and criminal Legislation under Tiberius, Middletown.

Rutledge, Steven, 2001, Imperial Inquisitions: Prosecutors and Informants from Tiberius to Domitian, London.

SAILOR, Dylan, 2008, Writing and Empire in Tacitus, New York.

Santalucia, Bernardo, 1994, Studi di diritto penale romano, Roma.

Schetrino, Maria Teresa, 2015, «L'attività edilizia di Augusto: memoria dell'Urbs e rappresentazione del potere», Paideia $L X X$, p. 353-372.

SYme, Ronald, 1997, Tacitus $I-I I, 2^{\text {a }}$ ed. (1 $1^{\text {a }}$ ed. 1958), Oxford.

WiLkInson, Samuel, 2012, Republicanism during the early Roman Empire, London - New York.

WISSE, Jakob, 2013, «Remembering Cremutius Cordus. Tacitus on History, Tiranny and Memory», Histos 7, p. $299-361$. 